\title{
Influence of Smartphone Usage on Low Back Pain and/or Shoulder Pain in College Students in a Judo Therapist Training Course: A pilot study
}

\author{
Yukio Yamamoto1)2), Nobuyuki Miyatake ${ }^{2)}$, Yutaka Owari12), Hiroaki Kataoka ${ }^{3)}$, \\ Akihiko Katayama4) \\ 1) Department of Judo Therapy, Shikoku Medical College, Utazu, Kagawa, 769-0205, Japan \\ Tel: +81-877-41-2323, Fax: +81-877-41-2321, Email: saiougauma@med.kagawa-u.ac.jp \\ 2) Department of Hygiene, Faculty of Medicine, Kagawa University, Miki, Kagawa 761-0952, \\ Japan \\ 3) Rehabilitation Center, KKR Takamatsu Hospital, Takamatsu, Kagawa 760-0018 Japan \\ 4) Department of Health and Sports Sciences, Shikoku Gakuin University, Zentsuji Kagawa \\ 765-8505, Japan
}

JAHS 10 (1): 1-9, 2019. Submitted Jan. 23, 2018. Accepted Aug. 20, 2018.

\begin{abstract}
The present study aimed to explore the effect of smartphone usage on low back pain (LBP) and shoulder pain (SP) in college students in a judo therapist course. From the course, 126 out of 155 students were enrolled in this cross-sectional study. Smartphone usage i.e. frequency, time and application purpose were evaluated using self-reported questionnaires. Clinical characteristics including LBP and SP were also evaluated. The prevalence of LBP and SP was 18 students (14.3\%) and 22 students (17.5\%) respectively. The usage time of smartphones in students with LBP or SP was significantly longer than in students without LBP or SP after adjustment for confounding factors. The prevalence of game usage in students with LBP was significantly higher than in students without. In addition, 2.5 hours for LBP and 5.0 hours for SP were the thresholds of usage time for smartphone by receiver operating characteristic (ROC) curve. Smartphone usage, especially usage time, was closely associated with LBP and SP. Proper usage of smartphones is needed for reducing LBP and SP in college students in the judo therapist course.
\end{abstract}

Key words: low back pain, shoulder pain, smartphone

\section{INTRODUCTION}

The number of smartphones is dramatically increasing and approximately $72.0 \%$ of the population were reported to be smartphone users in a WHITE PAPER Information and Communications in Japan by the Ministry of Internal Affairs and Communications ${ }^{1}$. Smartphone usage has many beneficial effects on our daily life and environment. However, 
some disadvantages, health related problems such as low vision ${ }^{2,3)}$, sleeplessness ${ }^{4,5)}$ and orthopedic disorders ${ }^{6 \sim 15)}$. In particular, low back pain (LBP) and shoulder pain (SP) are thought to be major problems ${ }^{12 \sim 15)}$.

LBP and SP are common disorders and have become a public health challenge in Japan ${ }^{16)}$. About $10.4 \%$ of Japanese have LBP and $8.8 \%$ have SP as reported by Ministry of Health, Labor and Welfare, Japan ${ }^{17)}$. Also, in occupational health problems, LBP is the most common disorder ${ }^{18)}$, and SP is well known to be associated with visual display terminal syndrome ${ }^{15}$. Also in Japanese students, LBP and/or SP are one of major health problems ${ }^{19}$. Therefore, prevention and management of LBP and SP are urgently required. Taken together, it is speculated that smartphone usage is closely linked to LBP and/or SP, and there are some reports about the link between smartphone usage and LBP and/or $\mathrm{SP}^{12 \sim 14)}$. However, there is no report evaluating the relationship between smartphone usage and LBP and/or SP in college students in a judo therapist course, who might be considered to be more health conscious of LBP and/or SP than other students. Because they specially learns judo therapy including musculoskeletal diseases in their school courses ${ }^{20}$ ).

Therefore, in this cross-sectional study, we investigated the effect of smartphone usage on LBP and/or SP in college students in a judo therapist course.

\section{MATERIALS AND METHODS}

\section{Subjects}

A total of 126 college students (107 men and 19 women), aged $26.0 \pm 8.8$ years, among 155 students, who met the entry criteria (Figure 1), were enrolled in this cross-sectional study.

The criteria were as follows: (1) Students in the judo therapist course, (2) Received self-reported questionnaires including smartphone usage, LBP and/or SP in November, 2016, (3) Not having acute pain of LBP and/or SP, and (4) Provided written informed consent.

Ethical approval was obtained from the ethical committee of Shikoku Medical College, Utazu, Japan (H26-3).

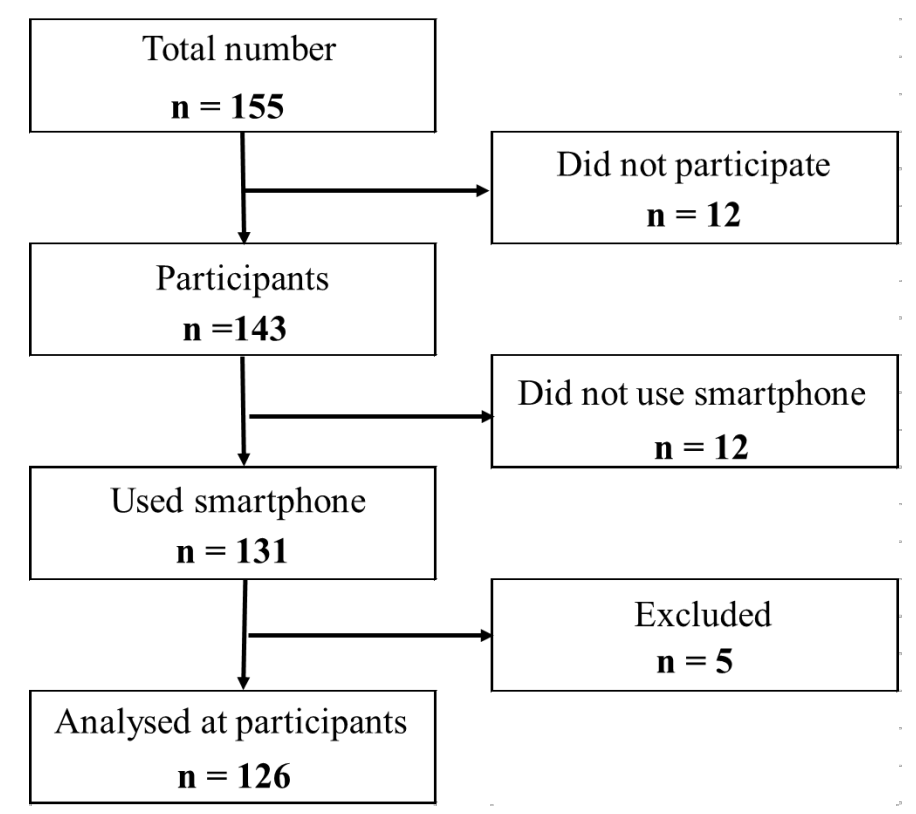

Figure 1 Flow diagram of analysis 


\section{Clinical parameters}

Clinical parameters, such as age, sex, height $(\mathrm{cm})$, body weight $(\mathrm{kg})$, body mass index (BMI), smartphone usage, psychological distress, exercise habits, LBP and SP, were obtained by self-reported questionnaires.

As parameters of smartphone usage, duration of usage (months), hours of usage per day, screen size of smartphone (inches), and data traffic of usage per month (gigabytes) were evaluated. We asked if the students used the smartphone daily for the following purposes even for a second i.e. social networking services (SNS), video watching, playing games, searching, viewing news, email, phone, listening to music, blogging or other uses. Body mass index (BMI) was calculated as follows: body weight $(\mathrm{kg}) /[\text { height }(\mathrm{cm})]^{2}$. Exercise habits (over the level of 30 minutes per week and the duration of one year) were also evaluated. Psychological distress was evaluated by K6 scores as previously described ${ }^{21}$.

LBP was evaluated as follows. "Do you have low back pain?" If the answer was "yes", they were classified as students with LBP. If the answer was "no", they were classified as students without LBP22). SP classification was the same as LBP classification.

\section{Statistical analysis}

All data were expressed as mean \pm SD. Comparison of parameters between subjects with and without LBP and/or SP was performed using un-paired t test and analyses of covariance (ANCOVA), where $p$ less than 0.05 was significant. The relationship between application purpose and LBP and/or SP was evaluated by $X$ square test. In addition, the threshold of usage time with LBP and/or SP was evaluated by receiver operating characteristic (ROC) curve. SPSS ver.22 for Windows software (IBM, Co. Ltd, Tokyo, Japan) was used for the analyses in this study.

\section{RESULTS}

Among the 155 students, 12 students did not participate in the questionnaire survey and 12 did not use a smartphone. 5 questionnaires were incomplete. Therefore, a total of 126 students are included in the analysis (Figure 1). The characteristics of the 126 students were shown in Table 1. Smartphone usage time per day was $3.1 \pm 2.4$ hours in men and $4.4 \pm 2.3$ hours in women.

Table 1 Clinical characteristics of enrolled subjects

\begin{tabular}{lccc}
\hline & Mean \pm SD & Minimum & Maximum \\
\hline Number of subjects & \multicolumn{4}{c}{126} \\
Men/women & $26.0 \pm 8.8$ & 18 & 56 \\
Age (years) & $168.6 \pm 7.4$ & 143.0 & 185.0 \\
Height (cm) & $68.8 \pm 14.9$ & 45.0 & 135.0 \\
Body weight (kg) & $24.1 \pm 4.3$ & 17.3 & 42.6 \\
Body mass index (kg/m $\left.{ }^{2}\right)$ & $43.9 \pm 18.5$ & 2.0 & 91.0 \\
Duration of usage (months) & $3.3 \pm 2.4$ & 0.3 & 15.0 \\
Hours of usage per day (hours) & $4.7 \pm 0.4$ & 3.7 & 6.0 \\
Screen size of smartphone (in.) & $5.2 \pm 3.4$ & 0.5 & 16.0 \\
Amount of usage per month (GB) & $4.1 \pm 4.3$ & 0 & 24 \\
K6 score &
\end{tabular}

GB: Gigabyte.

in. inch. 
The relationship between smartphone usage and LBP is shown in Table 2. There were $18(14.3 \%)$ students with LBP. Hours of smartphone usage per day were significantly longer in students with LBP than in students without LBP even after adjusting for age, sex, BMI and exercise habits. Comparisons between parameters in students with and without SP are shown in Table 3. Twenty-two students (17.5\%) had SP. Hours of smartphone usage per day and K6 scores were significantly higher in students with SP than in students without. Hours of smartphone usage per day in students with SP were significantly higher than in students without SP even after adjusting for age, sex, BMI and exercise habits.

In addition, we investigated the link between smartphone usage and sex, exercise habits and purpose. Playing games were significantly associated with LBP, and sex and exercise habits were also associated with SP (Table 4).

Finally, we investigated the thresholds of smartphone usage time for the occurrence of LBP and SP by ROC curve (Figure 2, 3). The threshold yielding the maximal sensitivity plus specificity for predicting LBP and SP was estimated to be 2.5 hours for LBP (Figure 2) and 5.0 hours for SP (Figure 3).

\section{DISCUSSION}

In this study, we firstly investigated the effect of smartphone usage on LBP and/or SP in college students in a judo therapist course in Japan. We found that the hours of smartphone usage per day was closely associated with LBP and/or SP, and 2.5 hours for LBP and 5.0 hours for SP were estimated as the thresholds of smartphone usage.

Hakala et al. reported neck-shoulder pain was perceived by $26 \%$ and LBP by $12 \%$ among 6,003 adolescents ${ }^{12}$. Shan et al. evaluated the prevalence of neck/shoulder pain as $48.4 \%$ and LBP as $33.1 \%$ among 3,600 students across 30 high schools in Shanghai ${ }^{13)}$.

In Japan, Yamada et al. presented that the prevalence of LBP was $25.2 \%$ by internet survey ${ }^{23)}$. The prevalence of neck-shoulder pain was $48.3 \%$ in Japanese general population ${ }^{24}$. In this study, the prevalence of LBP and SP was $14.3 \%$ and $17.5 \%$ in college students in a judo therapist course. The prevalence of LBP and SP seemed to be comparably low as we expected. The data obtained from this study provides us a useful database for the prevalence of LBP and/or SP in the college students in training courses for judo therapists in Japan.

Table 2 Comparison of parameters in subjects with and without low back pain

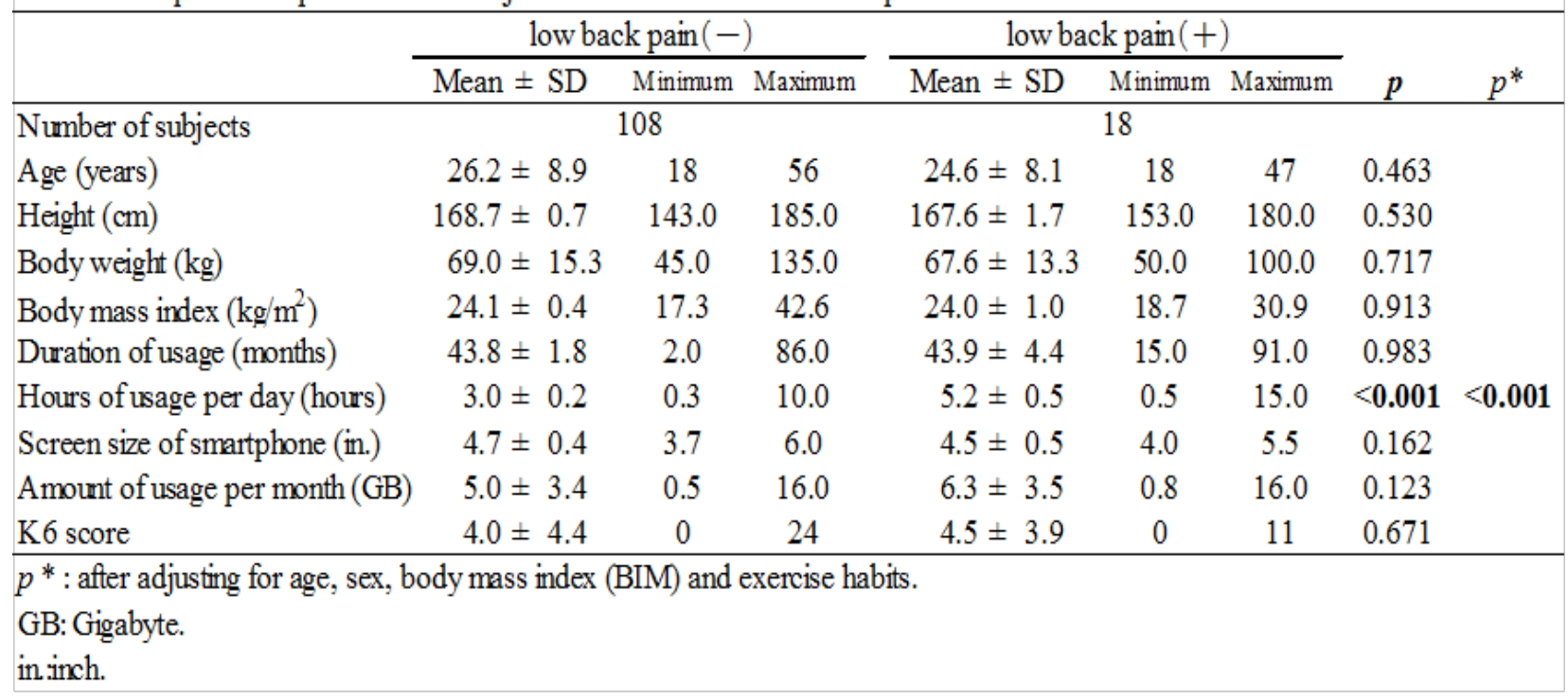


Table 3 Comparison of climical parameters in subjects with and without shoulder pain

\begin{tabular}{|c|c|c|c|c|c|c|c|c|}
\hline \multirow{3}{*}{ Number of subjects } & \multicolumn{3}{|c|}{ Shoulder pain $(-)$} & \multicolumn{3}{|c|}{ Shoulder pain $(+)$} & \multirow[b]{2}{*}{$p$} & \multirow[b]{2}{*}{$p^{*}$} \\
\hline & \multirow{2}{*}{ Mean \pm SD } & \multicolumn{2}{|c|}{ Minimum Maximum } & \multirow{2}{*}{ Mean \pm SD } & \multicolumn{2}{|c|}{ Minimum Maximum } & & \\
\hline & & 104 & & & 22 & & & \\
\hline Age (years) & $25.6 \pm 8.7$ & 18 & 56 & $27.5 \pm 9.5$ & 18 & 47 & 0.361 & \\
\hline Height (cm) & $168.9 \pm 7.2$ & 143.0 & 185.0 & $167.2 \pm 8.1$ & 153.0 & 180.0 & 0.349 & \\
\hline Body weight (kg) & $68.8 \pm 15.3$ & 45.0 & 135.0 & $68.6 \pm 13.3$ & 50.0 & 100.0 & 0.943 & \\
\hline Body mass index $\left(\mathrm{kg} / \mathrm{m}^{2}\right)$ & $24.0 \pm 4.4$ & 17.3 & 42.6 & $24.5 \pm 3.9$ & 18.9 & 32.0 & 0.666 & \\
\hline Duration of usage (months) & $44.0 \pm 1.8$ & 2.0 & 86.0 & $43.2 \pm 4.0$ & 22.0 & 91.0 & 0.862 & \\
\hline Hours of usage per day (hours) & $3.1 \pm 2.2$ & 0.3 & 15.0 & $4.3 \pm 3.2$ & 0.5 & 10.0 & 0.039 & 0.049 \\
\hline Screen size of smartphone (in) & $4.6 \pm 0.4$ & 3.7 & 6.0 & $4.7 \pm 0.4$ & 4.0 & 5.5 & 0.847 & \\
\hline Amount of usage per month (GB) & $5.2 \pm 3.5$ & 0.5 & 16.0 & $5.1 \pm 3.1$ & 0.8 & 10.0 & 0.919 & \\
\hline K6 score & $3.8 \pm 4.4$ & 0 & 24 & $5.6 \pm 3.6$ & 0 & 11 & 0.041 & 0.160 \\
\hline
\end{tabular}

$p^{*}$ : after adjusting for age, sex, body mass index (BMI) and exercise habits.

GB: Gigabyte.

in.inch.

Table 4 The relationship between daily smartphone usage and low back pain or shoulder pain

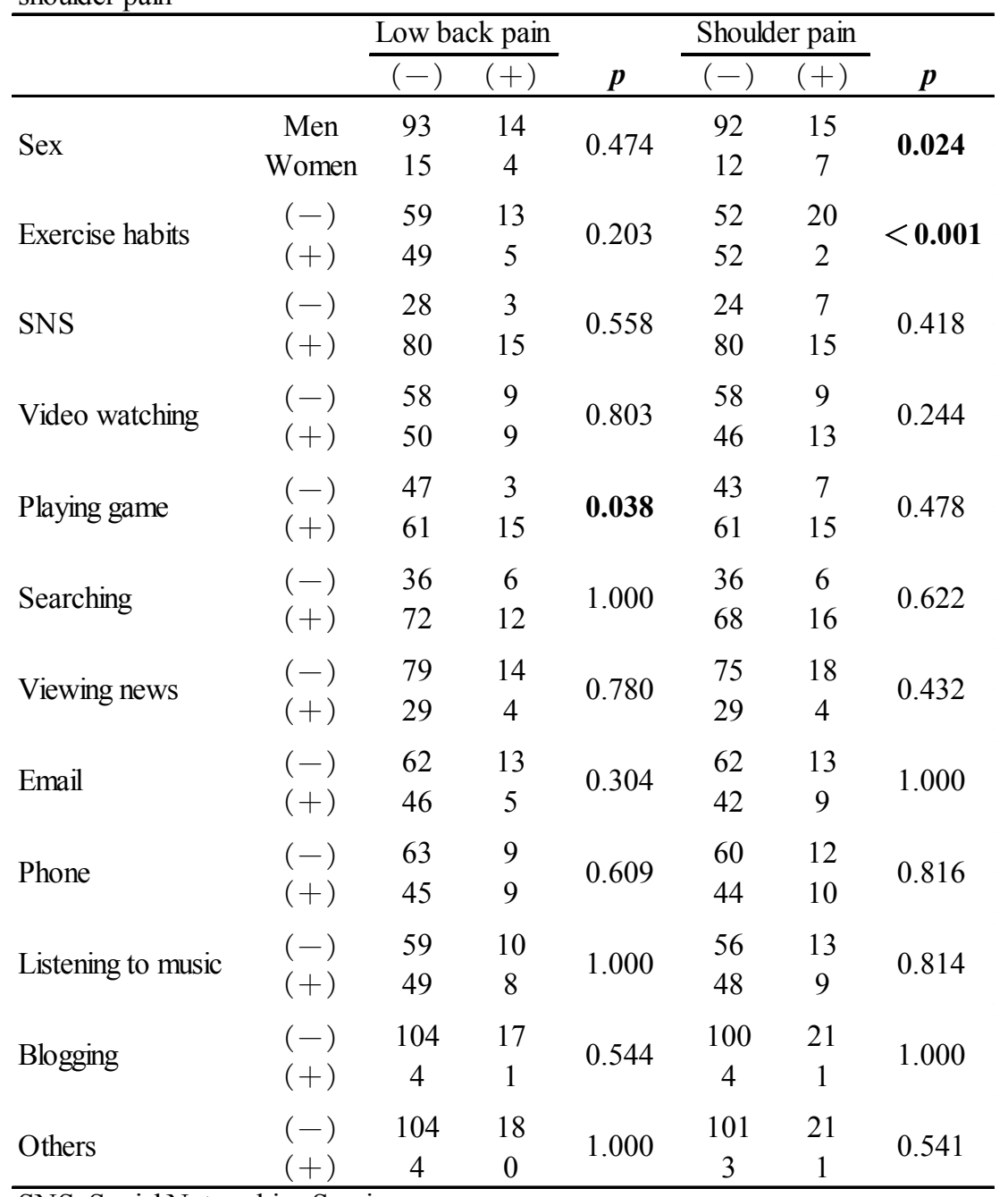

SNS: Social Networking Services. 


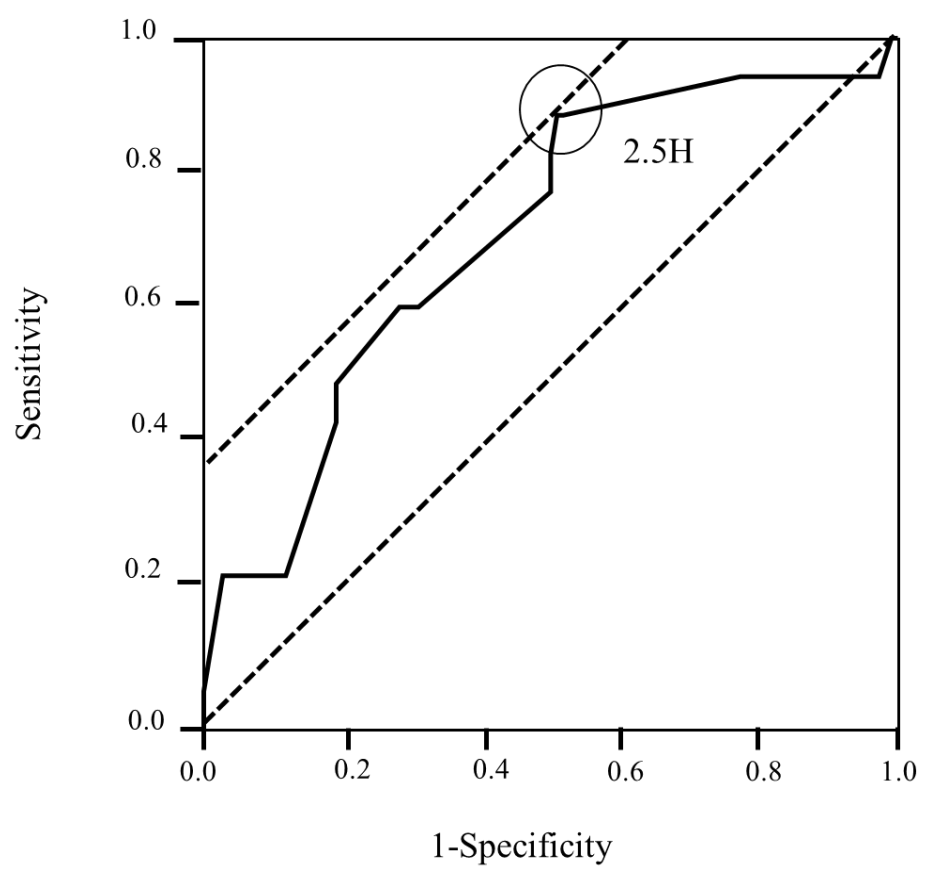

Sensitivity: 0.89, Specificity: 0.49, Area Under the Curve (AUC): 0.71

Figure 2 Receiver operating characteristic (ROC) curves of daily usage and low back pain

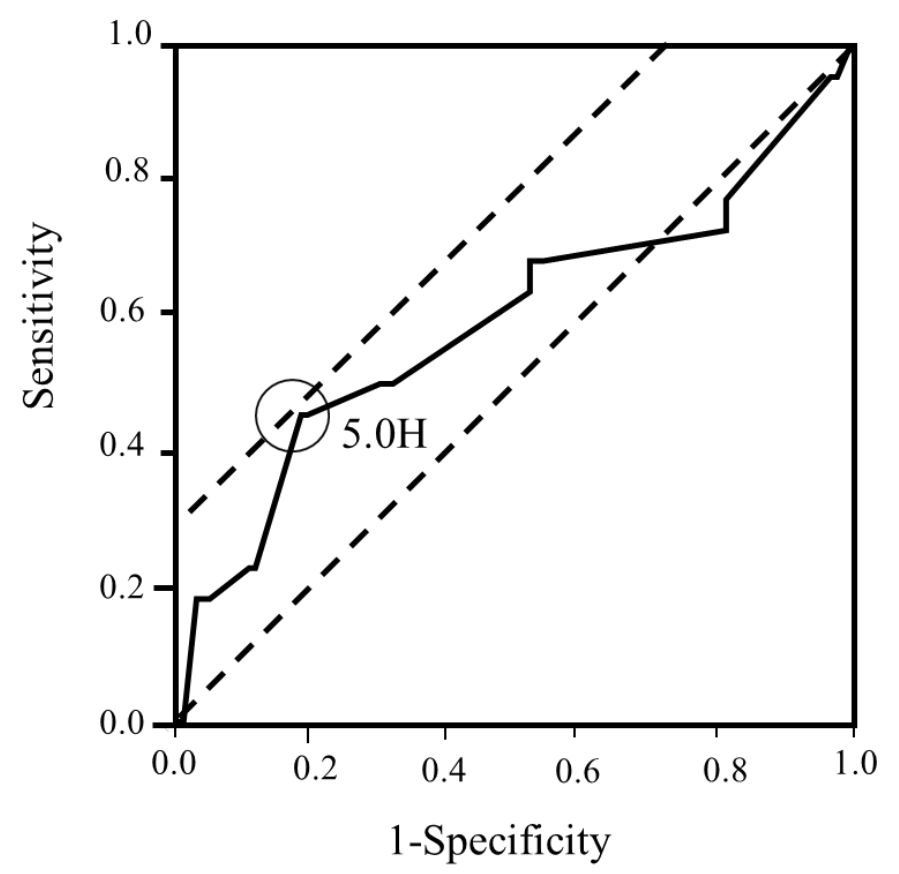

Sensitivity: 0.46, Specificity: 0.82, Area Under the Curve (AUC): 0.60

Figure 3 Receiver operating characteristic (ROC) curves of daily usage and shoulder pain 
According to the details of smartphone usage in relation to LBP and SP, Hakala et al. reported that daily use of computers exceeding 5 hours for LBP and 2-3 hours for neck-shoulder pain was a threshold ${ }^{12}$ ). Kim SY et al. also showed that pain and fatigue worsened with longer smartphone use ${ }^{25)}$. Yabe et al. long video-game-playing time per day was significantly associated with low back pain ${ }^{26)}$. Kim HJ et al. found that the area of the most painful body after smartphone usage was the shoulder and neck ${ }^{14)}$. However, Chiang et al. reported that found no significant difference between times spent using smartphone and musculoskeletal discomfort after smartphone usage ${ }^{27)}$. Although the relationship between smartphone usage and LBP and/or SP was partially controversial, we found that the hours of smartphone usage were closely associated with LBP and SP even after adjusting for confounding factors in both sexes.

The prevalence of SP was different between men and women. Playing games was associated with LBP, and exercise habits were associated with SP. These results indicate that the factor which is associated with LBP and/or SP might be different. In addition, smartphone usage at 2.5 hours for LBP and 5.0 hours for SP was estimated as a threshold. However, the thresholds obtained from this study did not agree with the report of Hakala et al., and we also did not find a relationship between smartphone screen size and LBP and/or SP. The enrolled students were thought to might be conscious of LBP and/or SP, and the sample size from one local college in Japan was small. These factors may affect the results of this study. However, reducing smartphone usage hours may prevent LBP and/or SP in college students in judo therapist training courses in clinical practice.

There are still some limitations in this study. Firstly, our study was cross-sectional, but not longitudinal. In addition, the duration of LBP and/or SP was not evaluated in this study. Therefore, we could not demonstrate a causal relationship between smartphone use and LBP and/or SP. Secondly, we could not clarify the underlying factors for the effect of smartphone usage on LBP and/or SP because we could not obtain detailed data. In other words, we could not evaluate whether the pain occurs at rest, and at smartphone use. We could not also evaluate the posture when they use their smartphone. In addition, the prevalence of SP in women was higher than that of SP in men as the previous report ${ }^{28)}$. Thirdly, the enrolled college students in our study were students at only one college, Japan; therefore, the results obtained from this study may not apply to all students in Japan. Nevertheless, it seems reasonable that reducing the usage time of smartphones and recommending their appropriate usage of smartphones would reduce LBP and/or SP in college students in the judo therapist training courses. Studies with a large sample and longitudinal design with detailed data are required.

\section{Conflicts of Interest}

All authors have no conflicts of interest to declare.

\section{REFERENCES}

1) Ministry of Internal Affairs and Communications. WHITE PAPER Information and Communications in Japan.

http://www.soumu.go.jp/johotsusintokei/ whitepaper/eng/WP2016/chapter-5.pdf\# page $=12$, accessed on Aug. 8, 2018.

2) Rosenfield M: Computer vision syndrome: a review of ocular causes and potential treatments. Ophthalmic Physiol Opt 31: 502-515, 2011.

3) Kim J, Hwang Y, Kang S et al: Association between Exposure to Smartphones and Ocular Health in Adolescents. Ophthalmic Epidemiol 23: 269-276, 2016. 
4) Demirci K, Akgönül $M$ and Akpinar $A$ : Relationship of smartphone use severity with sleep quality, depression, and anxiety in university students. J Behav Addict 4: 85-92, 2015.

5) Exelmans L and Van den Bulck J: Bedtime mobile phone use and sleep in adults. Soc Sci Med 148: 93-101, 2016.

6) Shin $\mathrm{H}$ and Kim K: Effects of Cervical Flexion on the Flexion-relaxation Ratio during Smartphone Use. J Phys Ther Sci 26: 1899-1901, 2014.

7) Kietrys DM, Gerg MJ, Dropkin J et al.: Mobile input device type, texting style and screen size influence upper extremity and trapezius muscle activity, and cervical posture while texting. Appl Ergon 50: 98-104, 2015.

8) Choi JH, Jung $\mathrm{MH}$ and Yoo KT: An analysis of the activity and muscle fatigue of the muscles around the neck under the three most frequent postures while using a smartphone. J Phys Ther Sci 28: 1660-1664, 2016.

9) Jung SI, Lee NK, Kang KW et al: The effect of smartphone usage time on posture and respiratory function. J Phys Ther Sci 28: 186-189, 2016.

10) Yang SY, Chen MD, Huang $Y C$ et al: Association Between Smartphone Use and Musculoskeletal Discomfort in Adolescent Students. J Community Health 42: 423-430, 2017.

11) Park JH Ms, Kang SY, Lee SG et al: The effects of smart phone gaming duration on muscle activation and spinal posture: Pilot study. Physiother Theory Pract 33: 661-669, 2017.

12) Hakala PT, Rimpelä AH, Saarni LA et al: Frequent computer-related activities increase the risk of neck-shoulder and low back pain in adolescents. Eur J Public Health 16: 536-541, 2006.

13) Shan Z, Deng G, Li J et al: Correlational analysis of neck/shoulder pain and low back pain with the use of digital products, physical activity and psychological status among adolescents in Shanghai. PLoS One 8: e78109, 2013.

14) Kim HJ and Kim JS: The relationship between smartphone use and subjective musculoskeletal symptoms and university students. J Phys Ther Sci 27: 575-579, 2015.

15) Iwakiri K, Mori I, Sotoyama $M$ et al: Survey on visual and musculoskeletal symptoms in VDT workers. J Occup Health 46: 201-212, 2004.

16) Hattori $\mathrm{S}$ : The prevalence of chronic pain in Japan. Folia Pharmacol Jpn 127: 176-180, 2006 (in Japanese).

17) Ministry of Health, Labour and Welfare. Comprehensive Survey of Living Conditions. http://www.mhlw.go.jp/toukei/saikin/hw/k-t yosa/k-tyosa16/dl/04.pdf, accessed on Aug. 8, 2018 (in Japanese)

18) Ministry of Health, Labour and Welfare. Survey on the occurrence of business conditions of disease.

http://www.mhlw.go.jp/bunya/roudoukijun/ anzeneisei11/dl/h28-10.pdf, accessed on Aug. 8, 2018 (in Japanese).

19) Kitsunai I, Otsuka Y: Investigation results of the appearance rate and the measures against stoop, lumbago and shoulder stiffness in university students. HUSCAP 104: 205-211, 2008.

20) Employment Security Bureau, Ministry of Health, Labour and Welfare. Hellowork Internet service.

https://www.hellowork.go.jp/doc2/B15301ju udouseifukusi.pdf, accessed on Aug. 8, 2018 (in Japanese).

21) Kessler RC, Barker PR, Colpe LJ, et al: Screening for serious mental illness in the general population. Arch Gen Psychiatry 60: 184-189, 2003.

22) Hishii S, Miyatake N, Nishi $\mathrm{H}$, et al: Psychological distress between chronic hemodialysis patients with and without 
low back pain. Environ Health Prev Med 21:487-491, 2016.

23) Yamada K, Matsudaira K, Takeshita K et al: Prevalence of low back pain as the primary pain site and factors associated with low health-related quality of life in a large Japanese population: a pain-associated cross-sectional epidemiological survey. Mod Rheumatol 24: 343-348, 2014.

24) Takasawa E, Yamamoto A, Kobayashi T et al: Characteristics of neck and shoulder pain in the Japanese general population. J Orthop Sci: 403-409, 2015.

25) Kim SY and Koo SJ: Effect of duration of smartphone use on muscle fatigue and pain caused by forward head posture in adults. J Phys Ther Sci 28: 1669-1672, 2016.

26) Yabe $Y$, Hagiwara Y, Sekiguchi T et al: Late bedtimes, short sleeping time, and longtime video-game playing are associated with low back pain in school-aged athletes. Eur Spine $J$ 28: 1112-1118, 2018.

27) Chiang HY and Liu CH: Exploration of the associations of touch-screen tablet computer usage and musculoskeletal discomfort. Work 53: 917-925, 2016.

28) Sarquis LM, Coggon D, Ntani G et al: Classification of neck/shoulder pain in epidemiological research: a comparison of personal and occupational characteristics, disability, and prognosis among 12,195 workers from 18 countries. Pain 157: 1028-1036, 2016. 